\title{
Decision-Aid Methods Based on Belief Function Theory with Application to Torrent Protection
}

\author{
Simon Carladous, Jean-Marc Tacnet, Jean Dezert, \\ and Mireille Batton-Hubert
}

\begin{abstract}
In mountainous areas, decision-makers must find the best solution to protect elements-at-torrential risk. The decision process involves several criteria and is based on imperfect information. Classical Multi-Criteria Decision-Aiding methods (MCDAs) are restricted to precise criteria evaluation for decision-making under a risky environment and suffer of rank reversal problems. To bridge these gaps, several MCDAs have been recently developed within belief function theory framework. The aims of this chapter are to introduce how these methods can be applied in practice and to introduce their general principles. To show their applicability to the real-life problem, we apply them to the Decision-Making Problem (DMP) comprising the comparison of several protective alternatives against torrential floods and selection of the most efficient one. We finally discuss the method improvements to promote their practical implementation.
\end{abstract}

Keywords Decision under uncertainty - Imperfect and conflicting information . Multicriteria decision analysis - Belief functions - Risk analysis and mitigation · Torrent protection

\footnotetext{
S. Carladous (殴

Département Risques Naturels (DRN), Office National des Forêts (ONF), Grenoble, France e-mail: simon.carladous@onf.fr

J.-M. Tacnet

Snow Avalanche Engineering and Torrent Control Research Unit (ETNA), Université Grenoble Alpes, Irstea - UR ETGR, St-Martin d'Hères Cedex, France e-mail: jean-marc.tacnet@irstea.fr

J. Dezert

ONERA, The French Aerospace Lab, Palaiseau Cedex, France

e-mail: jean.dezert@onera.fr

M. Batton-Hubert

Institut Henri Fayol, UMR LIMOS 6158, Ecole Nationale Supérieure des Mines de Saint-Etienne, Saint-Etienne Cedex 2, France

e-mail: mbatton@emse.fr; Mireille.BATTON-HUBERT@emse.fr
} 


\subsection{Introduction}

Mountainous torrential floods are different from plains' floods because of high flow velocity and high concentration of materials in flowing. Materials come from headwaters, are transported in a channel by debris flows or bedload transport, and finally spread on the alluvial fan. As shown in Fig. 15.1, they put people, buildings, and networks at risk.

Protective systems aim is to reduce damage on elements-at-risk. Therefore, they have specific functions. For instance, the check dam series maintain materials in headwaters whereas sediment traps stop materials before they reach elements-atrisk [1]. In practice, risk managers decide on actions based on several criteria, for example, cost vs. damage reduction. An example of a practical Decision-Making Problem (DMP) is given in Fig. 15.2. The goal is to compare several potential protective actions $A_{i}$ within a torrential watershed (i) to assign each alternative to a class (or label) as classically done by experts, or (ii) to rank all alternatives according to a preference order, or (iii) to choose the best alternative [2].

Classical Multi-Criteria Decision-Aiding methods (MCDAs) such as Analytic Hierarchy Process (AHP) [3], Technique for Order Preference by Similarity to the Ideal Solution (TOPSIS) [4], and Cost-Benefit Analysis (CBA) [5] help to make decision on such Multi-Criteria Decision-Making (MCDM) problems. While evaluations of criteria in practice are done with different units and scales, imperfect, provided by more or less reliable sources, and made under an epistemically uncertain environment [6], classical MCDAs only consider perfect criteria evaluation, suffer from rank reversal problems, and are limited to decisions under a risky environment.
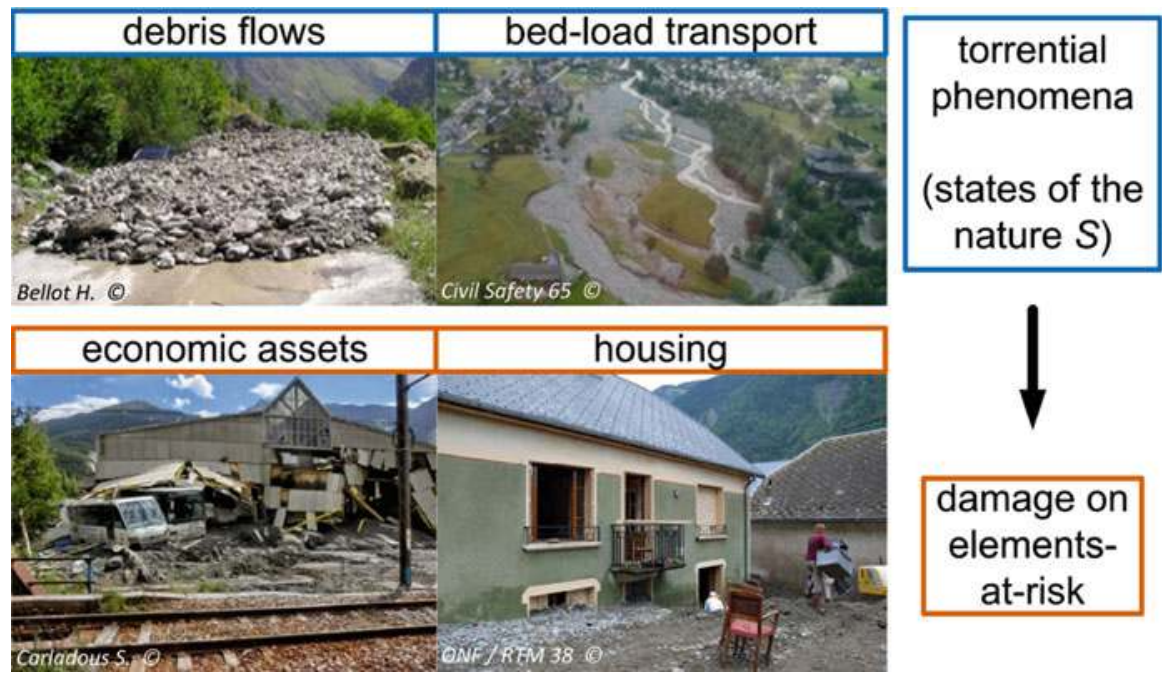

Fig. 15.1 Torrential phenomena and examples of elements-at-risk 
To bridge these gaps, several MCDAs were developed within belief function theory framework. As for any MCDA, the first step is to specify the DMP, potential alternatives, decision criteria [2], their scoring scale, and their importance weights. To assign each alternative to a qualitative label (i.e., good, bad, very bad, etc.), Evidential Reasoning for Multi-Criteria Decision Analysis (ER-MCDA) approach extends the AHP by taking into account imperfect evaluation of each criterion provided by several sources. Belief function theory is coupled [7] with fuzzy sets [8] and possibility theories [9]. Belief Function-based Technique for Order Preference by Similarity to the Ideal Solution (BF-TOPSIS) methods are more robust to rank reversal phenomena to rank all alternatives than classical MCDAs [10]. Cautious Ordered Weighted Averaging with Evidential Reasoning (COWA-ER) [11] and Fuzzy COWA-ER (FCOWA-ER) [12] improve initial OWA [13] to help to make decision under an epistemically uncertain environment.

This chapter shows how these new methods can be combined and applied in practice. Therefore, Sect. 15.2 not only recalls basics of MCDM problems and decision-making under uncertainty but also basics of fuzzy set, possibility, and belief function theories. Section 15.3 introduces general principles of ER-MCDA, BF-TOPSIS, and FCOWA-ER methods. They are then applied in Sect. 15.4 to the same DMP introduced in Fig. 15.2. In Sect. 15.5, we finally discuss needed improvements to encourage their practical implementation.

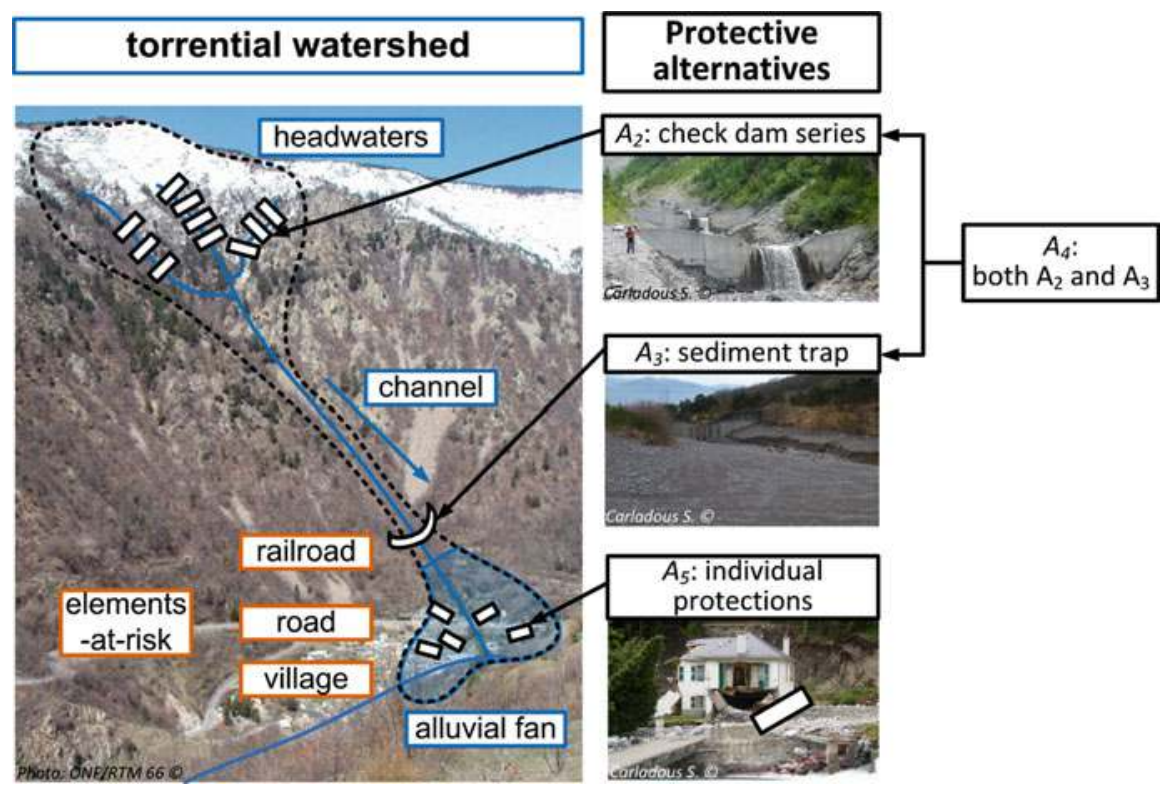

Fig. 15.2 A real-life DMP within a torrential watershed 


\subsection{Basics of Decision-Making and Imperfect Information}

This section provides a formal description of the DMPs, models of representing imperfect information, and decision methods given it.

\subsubsection{Formalization of Decision-Making Problems}

Any DMP is about comparing $M$ alternatives $A_{i} \in \mathscr{A}$ and selecting the best one. A decision-maker (DM) faces a MCDM problem if decision depends on several criteria $g_{j}, j=1, \ldots, N$. A set $\mathscr{S}$ represents the states of the nature. Since the beginning of the twentieth century, it has been proposed to distinguish decision-making under risk from decision-making under uncertainty, given the DM knowledge on $\mathscr{S}$ [14]. This subsection introduces related formalisms to represent the whole MCDM problem under uncertainty.

\subsubsection{Multi-criteria Decision-Making Problem}

A DM assigns an importance weight $\omega_{j}$ to each criterion $g_{j}, j=1, \ldots, N$. Respecting the condition $\sum_{j=1}^{N} \omega_{j}=1$, the vector $\mathbf{w}=\left[\omega_{1}, \ldots, \omega_{j}, \ldots, \omega_{N}\right]$ represents the DM preference over these criteria. For each $g_{j}$, a specific scoring scale $X_{j}$ is defined. The DM scores each alternative $A_{i}$ based on each $g_{j}$. This score is denoted $x_{i j} \in X_{j}$. The DM eventually provides the $M \times N$ score matrix $\mathbf{S}=\left[x_{i j}\right]$ defined by Eq. (15.1) [15].

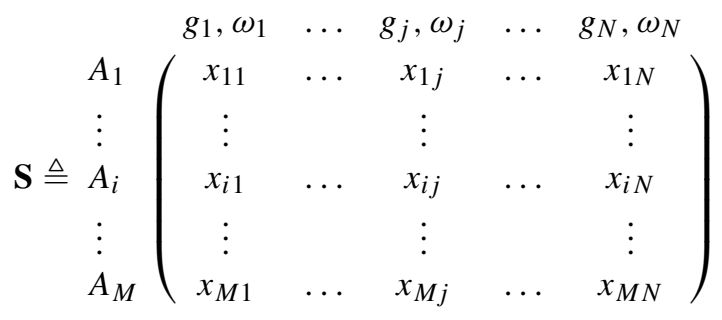

\subsubsection{Decision-Maker Preferences}

The DM has preferences not only over criteria but also to compare alternatives according to each criterion. First, AHP helps to establish the $N$-vector w which represents the DM preferences over criteria by comparing criteria pairwisely [3]. Second, DM has a preference ordering between all alternatives $A_{i} \in \mathscr{A}$, given a scoring scale $X_{j}$ for each $g_{j}$. For instance, considering three alternatives $A_{1}, A_{2}$, and $A_{3}$ and their scores $x_{1 j}, x_{2 j}, x_{3 j} \in X_{j}$, the DM preference can be represented by: 
- a total pre-order which assumes preference transitivity: if $A_{1} \succ A_{2}$ and $A_{2} \succ A_{3}$, then $A_{1} \succ A_{3}[16]$.

- a partial pre-order which relaxes transitivity assumption [2].

\subsubsection{From Decision-Making Under Risk to Decision-Making Under Uncertainty}

In practice, torrential hazard is generally represented by a finite set of states of the nature $\mathscr{S}=\left\{S_{1}, \ldots, S_{k}, \ldots, S_{K}\right\}$, as recalled in Fig. 15.1. Each $S_{k}$ is commonly referred to as scenario [17]. Given $S_{k}, C_{i k}$ is the global payoff expected for each alternative $A_{i}$. DM provides the $M \times K$ payoff matrix $\mathbf{C}=\left[C_{i k}\right]$ defined by Eq. (15.2) [11].

Given $\mathbf{C}$, decision-making depends on the DM knowledge on $\mathscr{S}[11]$ :

- Decision-making under certainty: since only one $S_{k}$ is known and certain to occur, it consists in choosing the best $A_{i^{\star}}$ with $i^{\star} \triangleq \arg \max _{i}\left\{C_{i k}\right\}$.

- Decision-making under risk (or aleatory uncertainty): the true state of the nature is unknown, but one knows all the probabilities $p_{k}=P\left(S_{k}\right)$. In the context of natural hazards, the expected payoff $E\left[C_{i}\right]=\sum_{k} p_{k} \cdot C_{i k}$ is generally computed for each $A_{i}$. The best $A_{i^{\star}}$ is with $i^{\star} \triangleq \arg \max _{i}\left\{E\left[C_{i}\right]\right\}$.

- Decision-making under ignorance: one assumes no knowledge about the true state of the nature but that it belongs to $\mathscr{S}$. Yager's Ordered Weighted Averaging (OWA) approach [13] can be used to make a decision in this context.

- Decision-making under uncertainty: a belief structure characterizes the knowledge on $S$. In practice, this is the closest representation of torrential hazard knowledge. Its elicitation by subjective probabilities $p_{k}=P\left(S_{k}\right)$ is usually used. Thus, decision-making is similar to decision-making under risk [18]. A more interesting approach is the OWA proposed by Yager [13] and improved in $[11,12]$.

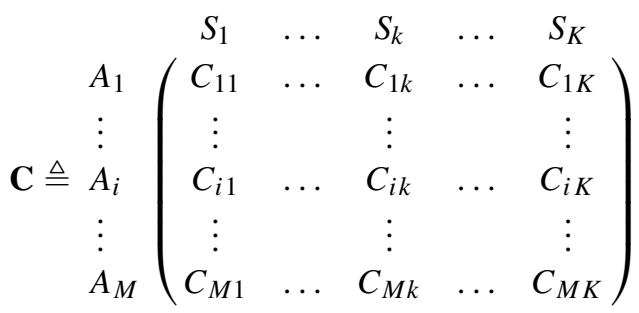

Formalisms of MCDM problems and decision-making under uncertainty are complementary in representing the DMP. A multi-criteria aggregation based on $\mathbf{S}$ can give each vector $\mathbf{C}_{\mathbf{k}} \triangleq\left[C_{1 k}, \ldots, C_{i k}, \ldots, C_{M k}\right]$ of $\mathbf{C}$. For each $A_{i}$ and $g_{j}$, computing the expected payoff $E\left[C_{i}\right]_{j}$ from $\mathbf{C}$ can provide $x_{i j}=E\left[C_{i}\right]_{j}$ in $\mathbf{S}$ [6]. 


\subsubsection{Several Types of Imperfect Information}

Whatever the DMP, decisions depend on the quality of information used to assess scores $x_{i j}(i=1, \ldots, M ; j=1, \ldots, N)$, payoffs $C_{i k}$, and states of the nature $S_{k}$ $(k=1, \ldots, K)$. There are various types of information imperfection [19]:

- inconsistency, which is related to conflict between sources such as several experts;

- imprecision referring, for example, to interval of numerical values;

- incompleteness, which represents the lack of information while data exist;

- aleatory uncertainty referring to aleatory events;

- epistemic uncertainty, which is linked to the lack of knowledge.

In practice, probabilities are usually used to represent imperfect information. A first criticism is their limit ${ }^{1}$ to represent uncertainty, while other formalisms are available: sets for imprecision, fuzzy sets for vagueness [8], possibility distributions, and imprecise probabilities for both uncertainty and imprecision [9, 27]. A second criticism is the use of subjective probabilities [18] both to decide under ignorance [20] and to represent the DM attitude with few information [21]. Belief function theory allows taking into account all types of imperfect information but also to make decisions under ignorance and epistemic uncertainty [22].

\subsubsection{What Is the Decision-Making Problem About?}

A DMP is about comparing the $M$ alternatives $A_{i}$ gathered in the set $\mathscr{A}$. In practice, three different objectives can be given [2]. For instance, the aim is to compare $M=4$ potential protective actions $A_{i}$ within a torrential watershed based on their efficiency:

1. to assign each $A_{i}$ to a predefined qualitative class (or label) of efficiency such as "high," "medium," "low," and "none" [23];

2. to rank all $A_{i}, i=1, \ldots, M$, totally or partially: for instance, $A_{3} \succ A_{4} \succ A_{1} \succ$ $A_{2}$ is a total order, while $A_{3} \sim A_{4} \succ A_{1} \sim A_{2}$ is a partial order;

3. to choose the best alternative $A_{i^{\star}} \in \mathscr{A}$, for instance, $A_{3}$.

\footnotetext{
${ }^{1}$ Indeed, the ignorance of a parameter value $x$ belonging to $[a, b]$ is usually modeled by a uniform probability distribution function (pdf) over $[a, b]$, which yields from the probability calculus to a nonuniform pdf of $1 / x$ on $[1 / b ; 1 / a]$. This result is not acceptable from the ignorance modeling standpoint because if one has no specific information on $x$, we cannot get more information on $1 / x$ but that $1 / x$ belongs to $[1 / b ; 1 / a]$. Therefore the uniform pdf often used to model ignorance in probability theory is problematic.
} 


\subsubsection{Imperfect Information: From Representation to Decision-Making}

This subsection details the three main steps to take into account imperfect information: (1) representation, (2) combination and propagation, and (3) decision [24].

\subsubsection{Representation of Imperfect Information Provided by a Source}

- Fuzzy set theory was developed to represent linguistic assessment of fuzziness [8]. Given individual elements $x$ of the universe of discourse $X$, the membership function $\mu_{\theta}(x) \in[0,1]$ associates each $x \in X$ to the fuzzy set $\theta$ with the grade of membership $\mu_{\theta}(x)$. As shown in Fig. 15.3a, a simple way to represent a membership function is to use a trapezoidal membership function defined by the quadruplet $\{a, b, c, d\}$ in Eq. (15.3) [25]: $[a, d]$ is the fuzzy set support denoted by $\operatorname{supp}_{\theta}$, while $[b, c]$ is its core $\operatorname{core}_{\theta}$. Given $X, \bar{\theta}$ is the complement fuzzy set of $\theta$ defined by Eq. (15.4) (Fig. 15.3a), and a mapping model [19] is a set $\Theta$ of $n$ fuzzy sets $\theta_{e}$, for $e=1, \ldots, n$ (Fig. 15.3b). Given two fuzzy sets $\theta_{1}$ and $\theta_{2}$, the membership function $\mu_{\theta_{1} \cup \theta_{2}}$ defined by Eq. (15.5) represents their union (Fig. 15.3c), while their intersection $\mu_{\theta_{1} \cap \theta_{2}}$ is defined by Eq. (15.6) [8].

$$
\begin{gathered}
\begin{cases}0 & \text { if } x \notin \text { supp }_{\theta} \\
\frac{x-a}{b-a} & \text { if } x \in[a, b] \\
1 & \text { if } x \in \text { core }_{\theta} \\
\frac{x-d}{c-d} & \text { if } x \in[c, d]\end{cases} \\
\mu_{\bar{\theta}}(x) \triangleq 1-\mu_{\theta}(x), x \in X \\
\mu_{\theta_{1} \cup \theta_{2}}(x) \triangleq \max _{x \in X}\left(\mu_{\theta_{1}}(x), \mu_{\theta_{2}}(x)\right) \\
\mu_{\theta_{1} \cap \theta_{2}}(x) \triangleq \min _{x \in X}\left(\mu_{\theta_{1}}(x), \mu_{\theta_{2}}(x)\right)
\end{gathered}
$$

a b

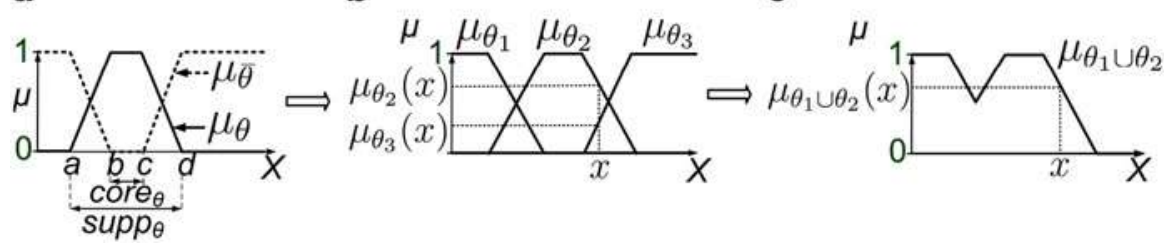

Fig. 15.3 (a) Fuzzy set $\Theta$ and its complement $\bar{\Theta}$; (b) mapping model $(n=3)$; (c) union 


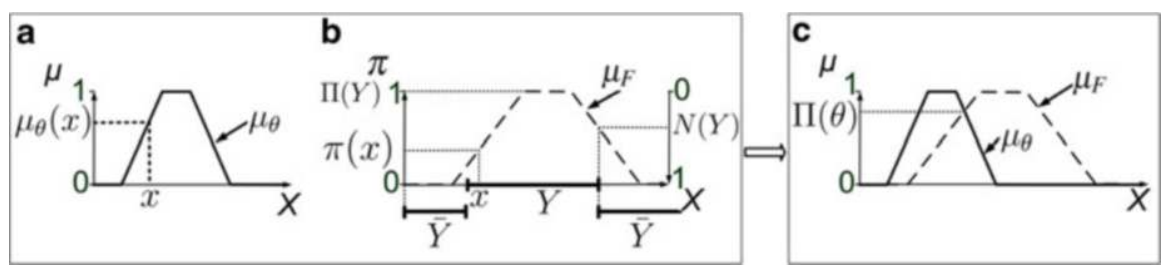

Fig. 15.4 (a) Fuzzy set $\Theta$; (b) possibility distribution $\pi=\mu_{F}$; (c) possibility measure of $\Theta$ given $\pi$

- Zadeh, Dubois, and Prade then developed the possibility theory in the fuzzy logic framework [9, 27]. Considering the fuzzy set $F$ of possible values of $x \in X$, the possibility distribution $\pi$ is given by $\mu_{F}(x) \triangleq \pi(x) \in[0,1]$ [26]. Given $Y$ a subset of $X$ and $\bar{Y}$ its complement, the possibility and necessity measures are $\Pi(Y) \triangleq \sup _{x \in Y} \pi(x)$ [9] and $N(Y) \triangleq 1-\Pi(\bar{Y}), \forall Y, \bar{Y} \subseteq X$ [27], as shown in Fig. 15.4b. $\Pi(Y)$ and $N(Y)$ are considered as the upper and lower bounds of the probability $P(Y)$. Given $X$, the membership function $\mu_{\theta}$, and a possibility distribution $\mu_{F}$ (Fig. 15.4c), the possibility measure of $\theta$ denoted by $\Pi(\theta)$ is defined by Eq. (15.7) [9].

$$
\Pi(\theta) \triangleq \sup _{x \in X} \mu_{\theta \cap F}(x)
$$

- In the meantime, Shafer introduced the belief function theory, also called Dempster-Shafer Theory (DST) [22]. The Frame of Discernment (FoD) is a finite set $\Theta=\left\{\theta_{1}, \ldots, \theta_{e}, \ldots, \theta_{n}\right\}$, with $n>1$, which gathers the potential answers of the DMP under concern. In DST, all FoD elements are assumed exhaustive and mutually exclusive. The power set of $\Theta$ denoted by $2^{\Theta}$ is the set of all subsets of $\Theta$, the empty set $\varnothing$ included. The complement of a subset $A \in 2^{\Theta}$ is denoted $\bar{A}$. Its cardinality is $|A|$. A source (or body) of evidence is characterized by a basic belief assignment $(\mathrm{BBA}) m^{\Theta}(\cdot)$, which is a mapping $m^{\Theta}(\cdot): 2^{\Theta} \rightarrow[0,1]$ that satisfies $m^{\Theta}(\emptyset)=0$, and $\forall A \neq \emptyset \in 2^{\Theta}$ the condition $\sum_{A \subseteq \Theta} m^{\Theta}(A)=1$. The vacuous BBA models the full ignorance of the source of evidence. If $m^{\Theta}(A)>0$, $A$ is a focal element of $m^{\Theta}(\cdot) . m_{A}^{\Theta}$ denotes the categorical BBA which focuses on $A \neq \emptyset$. More precisely, $m_{A}^{\Theta}(A)=1$ and $m_{A}^{\Theta}(Y)=0$ for any $Y \neq A$. Focal elements of a Bayesian BBA are only singletons on $2^{\Theta}$. Given the FoD $\Theta$ for final decision, it is possible to represent imperfect evaluation of the score $x_{i j}$, for each alternative $A_{i}$ according to each criterion $g_{j}$, through the BBA $m_{i j}^{\Theta}(\cdot)$ providing the $M \times N$ BBA matrix $\mathbf{M}^{\Theta}=\left[m_{i j}^{\Theta}(\cdot)\right]$. Thus, it must be compared with the $M \times N$ score matrix $\mathbf{S}=\left[x_{i j}\right]$, defined by Eq. (15.1). Given $m^{\Theta}(\cdot)$, belief and plausibility functions are, respectively, defined by: 


$$
\begin{gathered}
\operatorname{Bel}^{\Theta}(A) \triangleq \sum_{Y \subseteq A \mid Y \in 2^{\Theta}} m^{\Theta}(Y) \\
\operatorname{Pl}^{\Theta}(A) \triangleq \sum_{Y \cap A \neq \emptyset \mid Y \in 2^{\Theta}} m^{\Theta}(Y)
\end{gathered}
$$

Considering that the universe of discourse $X$ is the FoD $\Theta$, the plausibility measure $\mathrm{Pl}^{\Theta=X}(A)$ is a possibility measure $\Pi(A), \forall A \subseteq \Theta=X[26]$. $\operatorname{Bel}^{\Theta}(A)$ and $\mathrm{Pl}^{\Theta}(A)$ are interpreted as lower and upper bounds of the unknown probability $\mathrm{P}^{\Theta}(A)$. The interval $\mathrm{BI}^{\Theta}(A) \triangleq\left[\mathrm{Bel}^{\Theta}(A), \mathrm{Pl}^{\Theta}(A)\right]$ is its belief interval. Its length $\mathrm{Pl}^{\Theta}(A)-\mathrm{Bel}^{\Theta}(A)$ characterizes the uncertainty, also called ambiguity, on $A$ [28].

Given $m^{\Theta}(\cdot)$, several transformations help to approximate probability function $\mathrm{P}^{\Theta}(\cdot)$ : Smets' pignistic transformation provides $\operatorname{BetP}^{\Theta}(\cdot)$ [29], DSmP transformation gives $\operatorname{DSmP}_{\epsilon}^{\Theta}(\cdot)$ where $\epsilon \geq 0$ is a tuning parameter [30] (Vol. 3), and others.

Shafer's exhaustivity assumption means that the FoD is considered as a "closed world" (c.w.). In some practical problems, this assumption is too strict and it is more convenient to consider the original FoD as an "open world" (o.w.).

1. In Smets' Transferable Belief Model (TBM) [31], $\Theta^{\text {o.w. }} \triangleq\left\{\theta_{1}, \ldots, \theta_{q}\right\}$ and $\varnothing=$ $\bar{\Theta}^{\text {o.w. }}$. One has $\sum_{A \in 2^{\Theta}} m(A)=1$, and one allows $m(\emptyset) \geqslant 0$.

2. In Yager's approach [32], the open world is closed by an hedge element $\theta^{c}$, so that $\Theta^{\text {c.w. }} \triangleq \Theta^{\text {o.w. }} \cup\left\{\theta^{c}\right\}$. The cardinality $\left|\theta^{c}\right|$ is not known.

Shafer's mutual exclusivity assumption can be also too strict. DezertSmarandache Theory (DSmT) framework modifies DST to relax this assumption and proposes new techniques to combine the sources of evidence and to make a decision [30].

\subsubsection{Combining Information Provided by Several Sources of Evidence}

First of all, the source reliability and its importance must be clearly distinguished. Reliability is the source objective ability to give the correct solution of the DMP [33]. For each source $s_{q}, q=1, \ldots, Q$, it is represented by a reliability discounting factor $\alpha_{q} \in[0,1]$ [34]. Given the initial BBA $m_{q}^{\Theta}(\cdot)$ provided by $s_{q}$, Shafer's discounting method defined by Eq. (15.10) is generally used to provide the discounted mass $m_{\alpha_{q}}^{\Theta}(A)[22]$.

$$
m_{\alpha_{q}}^{\Theta}(A) \triangleq \begin{cases}\alpha_{q} \cdot m_{q}^{\Theta}(A) & \text { if } A \in 2^{\Theta} \neq \Theta \\ \alpha_{q} \cdot m_{q}^{\Theta}(A)+\left(1-\alpha_{q}\right) & \text { if } A=\Theta\end{cases}
$$


Importance is the subjective weight granted to the source by DM [33]. In a MCDM problem, each criterion can be considered as a source represented by a BBA $m_{j}^{\Theta}(\cdot)$. Each weight $\omega_{j}$ is the importance discounting factor used to provide the discounted $\mathrm{BBA}^{2} m_{\omega_{j}}^{\Theta}(\cdot)$.

$$
m_{\omega_{j}}^{\Theta}(A) \triangleq \begin{cases}\omega_{j} \cdot m_{j}^{\Theta}(A) & \text { if } A \in 2^{\Theta} \neq \emptyset \\ \omega_{j} \cdot m_{j}^{\Theta}(A)+\left(1-\omega_{j}\right) & \text { if } A=\emptyset\end{cases}
$$

Once BBAs have been discounted, the combination of distinct sources of evidence is denoted by $\oplus$ to provide the combined BBA $m_{\oplus}^{\Theta}(A), A \subset \Theta$. The largely used initial Dempster's rule $(D S)$ [22] has been subject to strong debates in fusion community, showing it does not behave well in high conflicting case [35] but also in low conflicting cases [36].

As a consequence, since the 1990s, many alternatives have been proposed to combine belief functions more or less efficiently. The Proportional Conflict Redistribution (PCR) rules have been developed in DSmT [30] (Vol. 3) to palliate disadvantages of the classical Dempster's fusion rule [37]. PCR rule $n^{\circ} 6$ (PCR6) defined by Eq. (15.12) for combining two sources of evidence $(K=2) m_{1}^{\Theta}(\cdot)$ and $m_{2}^{\Theta}(\cdot)$ is also consistent for more than two bodies of evidence $(K>2)$ [38].

$$
\begin{aligned}
& m_{\mathrm{PCR} 6}^{\Theta}(A) \triangleq \sum_{\substack{X_{1}, X_{2} \in 2^{\Theta} \\
X_{1} \cap X_{2}=A}} m_{1}^{\Theta}\left(X_{1}\right) \cdot m_{2}^{\Theta}\left(X_{2}\right) \\
&+\sum_{\substack{Y \in 2^{\Theta} \backslash\{A\} \\
A \cap Y=\emptyset}}\left[\frac{m_{1}^{\Theta}(A)^{2} \cdot m_{2}^{\Theta}(Y)}{m_{1}^{\Theta}(A)+m_{2}^{\Theta}(Y)}+\frac{m_{2}^{\Theta}(A)^{2} \cdot m_{1}^{\Theta}(Y)}{m_{2}^{\Theta}(A)+m_{1}^{\Theta}(Y)}\right]
\end{aligned}
$$

Combination by PCR6 fusion rule of the $N$ importance discounted BBAs $m_{j}^{\Theta}(\cdot)$ defined by Eq. (15.11) provides a BBA denoted $m_{\mathrm{PCR} 6_{\emptyset}}^{\Theta}(\cdot)$ with $m_{\mathrm{PCR} 6_{\emptyset}}^{\Theta}(\emptyset)>0$. Then, we commit zero to the mass of the empty set, and we normalize this BBA to get a proper normalized BBA $m_{\mathrm{PCR} 6}^{\Theta}(\cdot)$ with $m_{\mathrm{PCR} 6}^{\Theta}(\emptyset)=0$; see [33] for details.

\subsubsection{Decision-Making Given a Combined Belief Mass}

Given a BBA $m^{\Theta}(\cdot)$, choosing a singleton $\hat{\theta} \in \Theta$ or a subset $\hat{A} \subseteq \Theta$ is the decision issue. In general, it consists in choosing $\hat{\theta}=\theta_{e^{\star}}, e=1, \ldots, n$ with $e^{\star} \triangleq \arg \max _{e} C\left(\theta_{e}\right)$, where $C\left(\theta_{e}\right)$ is a decision-making criterion chosen according

\footnotetext{
${ }^{2}$ For a technical reason, one allows to commit some mass on the empty set in this discounting. This is not a problem because the final fusion result will be normalized.
} 
to the DM attitude: belief for a pessimistic attitude, plausibility for an optimistic one, one of the probabilistic transformations for an attitude of compromise.

In general, the DM attitude is not well known in DST. Moreover, in some practical cases, taking into account non-singletons $A \subseteq \Theta$ is needed to decide. For these cases, the minimum of any strict distance metric $d\left(m^{\Theta}, m_{A}^{\Theta}\right)$ between $m^{\Theta}(\cdot)$ and the categorical BBA $m_{A}^{\Theta}(\cdot)$ can be used in Eq. (15.13) [39]. If only singletons of $2^{\Theta}$ are accepted, decision is defined by Eq. (15.14).

$$
\begin{aligned}
& \hat{A}=\arg \min _{A \in 2^{\Theta}} d_{\mathrm{BI}}\left(m^{\Theta}, m_{A}^{\Theta}\right) \\
& \hat{\theta}=\arg \min _{\theta_{e} \in \Theta} d_{\mathrm{BI}}\left(m^{\Theta}, m_{\theta_{e}}^{\Theta}\right)
\end{aligned}
$$

Among the few true distance metrics ${ }^{3}$ between two BBAs $m_{1}^{\Theta}(\cdot)$ and $m_{2}^{\Theta}(\cdot)$, the belief interval-based Euclidean $d_{B I}\left(m_{1}^{\Theta}, m_{2}^{\Theta}\right) \in[0,1]$ is based on Wasserstein's distance [40] and provides reasonable results [41].

The quality indicator $q(\hat{A})$ defined by Eq. (15.15) evaluates how good the decision $\hat{A}$ is with respect to other focal elements: the higher $q(\hat{A})$ is, the more confident DM should be in its decision $\hat{A}$. If only singletons of $2^{\Theta}$ are accepted, $q(\hat{\theta})$ is defined by Eq. (15.16).

$$
\begin{aligned}
& q(\hat{A}) \triangleq 1-\frac{d_{\mathrm{BI}}\left(m^{\Theta}, m_{A}^{\Theta}\right)}{\sum_{A \in 2^{\Theta} \backslash\{\emptyset\}} d_{\mathrm{BI}}\left(m^{\Theta}, m_{A}^{\Theta}\right)} \\
& q(\hat{\theta}) \triangleq 1-\frac{d_{\mathrm{BI}}\left(m^{\Theta}, m_{\theta_{e}}^{\Theta}\right)}{\sum_{e=1}^{n} d_{\mathrm{BI}}\left(m^{\Theta}, m_{\theta_{e}}^{\Theta}\right)}
\end{aligned}
$$

\subsection{Belief Function-Based Decision-Aiding Methods}

Classical Decision-Aiding Methods (DAMs) have some limitations: (i) classical MCDAs do not consider imperfect evaluations of criteria, (ii) ranking can be affected by rank reversal problems [42, 43], and (iii) probability framework is limited by an epistemic uncertainty affecting the knowledge on the states of the nature $\mathscr{S}[20,21]$. This section introduces new belief function-based DAMs which help to overcome these three limitations using (i) Evidential Reasoning for MultiCriteria Decision Analysis (ER-MCDA) [44], (ii) Belief Function-based Technique for Order Preference by Similarity to Ideal Solution (BF-TOPSIS) methods [39], and (iii) Fuzzy Cautious Ordered Weighted Averaging with Evidential Reasoning (FCOWA-ER) [12], respectively.

\footnotetext{
${ }^{3}$ For any BBAs $x, y, z$ defined on $2^{\Theta}$, a true distance metric $d(x, y)$ satisfies the properties of nonnegativity $(d(x, y) \geq 0)$, non-degeneracy $(d(x, y)=0 \Leftrightarrow x=y)$, symmetry $(d(x, y)=d(y, x))$, and triangle inequality $(d(x, y)+d(y, z) \geq d(x, z))$.
} 


\subsubsection{ER-MCDA: Multi-criteria Assignment Given Imperfect Scores}

As detailed in Fig. 15.5, ER-MCDA methodology [44] is an extension of the Analytic Hierarchy Process (AHP) [3]. Given the FoD for decision $\Theta$ (step 1), it associates fuzzy logic framework and belief function theory to represent imperfect evaluation of the score of each alternative $A_{i}, i=1, \ldots, M$ based on each criterion $g_{j}, j=1, \ldots, N$, potentially provided by several sources $s_{q}, q=1, \ldots, Q$, through a BBA $m_{q, i j}^{\Theta}(\cdot)$ (step 2). A second improvement is the combination of BBAs taking into account reliability $\alpha_{q}$ of each source and importance $\omega_{j}$ of each criterion $g_{j}$ (step 3). It finally helps to assign each $A_{i}$ to the element $\hat{\theta}\left(A_{i}\right)$ (step 4).

\subsubsection{ER-MCDA-Step 1: DMP Formalization}

The scoring scale $X_{j}$ is specified for each criterion $g_{j}, j=1, \ldots, N$. The FoD for decision $\Theta$ is also defined: for instance, we consider four qualitative labels (or classes) $\theta_{e}$ of efficiency with $\Theta=\left\{\theta_{1}=\right.$ high, $\theta_{2}=$ medium, $\theta_{3}=$ low, $\theta_{4}=$ none\}.

\subsubsection{ER-MCDA-Step 2: BBA $m_{q, i j}^{\Theta}(\cdot)$ Construction}

For each $g_{j}, j=1, \ldots, N$, the mapping model is first provided (Fig. 15.3): $n$ fuzzy sets $\mu_{j, \theta_{e}}$ represent a partial pre-order of the DM preference for $X_{j}$ [44]. Then, for each alternative $A_{i}, i=1, \ldots, M$, each source (e.g., an expert) $s_{q}$ provides its imprecise and uncertain evaluation of $x_{i j} \in X_{j}$ through a possibility distribution $\pi_{q, i j}$ (Fig. 15.4).

Given these elements, the mapping process consists in transforming each $\pi_{q, i j}$ into a BBA $m_{q, i j}^{\Theta}(\cdot)$ using the $g_{j}$ mapping model. The initial mapping process [19] was based on a geometric transformation and was restricted to provide only Bayesian BBAs. A new mapping model was recently developed to provide general BBAs [45, 46], for each $A_{i}$ and each $g_{j}$ :

1. since fuzzy sets are given for an open world, Yager's hedged element $\theta^{c}$ [32] is used to provide membership functions in an hedged world (c.w.), and all membership functions $\mu_{j, X}, X \neq \emptyset \in \Theta$ are built applying Eq. (15.5);

2. all membership functions $\mu_{j, \bar{X}}, X \neq \emptyset \in \Theta$ are built applying Eq. (15.4);

3. given the possibility distribution $\pi_{q, i j}$, Eq. (15.7) gives the possibility measures $\Pi_{q, i j}(\bar{X})$ corresponding to the plausibility measure $\mathrm{Pl}_{q, i j}^{\Theta}(\bar{X})$;

4. the belief function $\operatorname{Bel}_{q, i j}^{\Theta}(\cdot)$ is directly obtained such as the BBA $m_{q, i j}^{\Theta}(\cdot)$. 


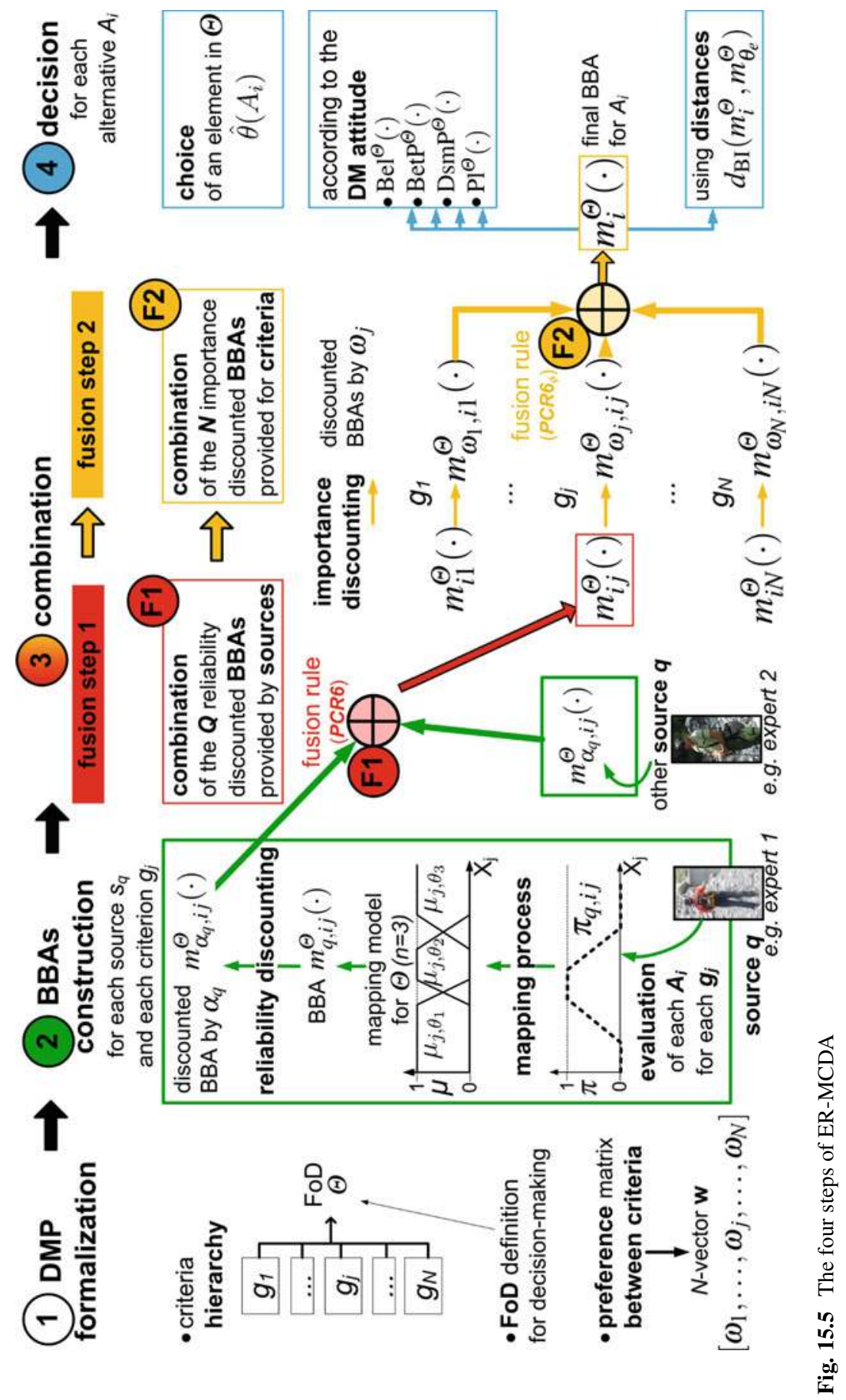




\subsubsection{ER-MCDA-Step 3: Combination of Two BBAs}

For each $A_{i}$ and each $g_{j}$, fusion 1 combines BBAs provided by $Q$ sources $s_{q}$, taking into account reliability factor $\alpha_{q}$ and using PCR6 fusion rule. For each $A_{i}$, fusion 2 combines BBAs provided for $N$ criteria $g_{j}$, taking into account importance factor $\omega_{j}$ and using PCR6 $6_{\emptyset}$ fusion rule. Final BBA $m_{i}^{\Theta}(\cdot)$ is obtained.

\subsubsection{ER-MCDA-Step 4: Making Decision for Each $A_{i}$}

Given $\Theta$ and $m_{i}^{\Theta}(\cdot)$, DM must assign each $A_{i}$ to labels by choosing $\hat{\theta}\left(A_{i}\right)$. Therefore, DM can decide according to a pessimistic attitude (max of belief), an optimistic one (max of plausibility), or an attitude of compromise (max of subjective probability).

For the latter, whatever the probability transformation, the cardinality $\left|\theta^{c}\right|$ must be known. Therefore, a strong hypothesis is to consider $\left|\theta^{c}\right|=1$ which can be theoretically discussed. Using Eq. (15.14) to decide through the minimal of belief interval distance does not involve any hypothesis on it.

\subsubsection{BF-TOPSIS: A More Robust Multi-criteria Ranking}

Given the score matrix $\mathbf{S}$ defined by Eq. (15.1), the classical MCDAs such as the AHP [3], Technique for Order Preference by Similarity to the Ideal Solution (TOPSIS) [4], or Estimator Ranking Vector (ERV) [47] are limited by rank reversal problems [42, 43]. As detailed in Fig. 15.6, the four new BF-TOPSIS methods [10] are inspired by the ERV to avoid a normalization step and by TOPSIS to compare each $A_{i}$ with an ideal best and an ideal worst solutions. They are based on a common preliminary step and have an increasing computation complexity and robustness to rank reversal problems $[6,10]$.

\subsubsection{Preliminary Step: DMP Formalization and BBA $m_{i j}^{\mathscr{A}}(\cdot)$ Construction}

A DMP is about ranking all alternatives $A_{i}$ and choosing the best one $A_{i^{\star}} \in \mathscr{A}$ : the FoD $\Theta$ for decision is the set of alternatives $\mathscr{A}$. Given the score matrix $\mathbf{S}$ (see Fig. 15.6), this common step consists of constructing the $M \times N$ BBA matrix $\mathbf{M}^{\mathscr{A}}=$ $\left[m_{i j}^{\mathscr{A}}(\cdot)\right]$.

For each $A_{i}$ and $g_{j}$, the positive support $\operatorname{Sup}_{j}\left(A_{i}\right) \triangleq \sum_{k \in\{1, \ldots, M\} \mid x_{k j} \leq x_{i j}} \mid x_{i j}-$ $x_{k j} \mid$ and the negative one $\operatorname{Inf}_{j}\left(A_{i}\right) \triangleq-\sum_{k \in\{1, \ldots, M\} \mid x_{k j} \geq x_{i j}}\left|x_{i j}-x_{k j}\right|$, respectively, measure how much $A_{i}$ is better and worse than other alternatives according to $g_{j}$. Given $A_{\max }^{j} \triangleq \max _{i} \operatorname{Sup}_{j}\left(A_{i}\right)$ and $A_{\min }^{j} \triangleq \min _{i} \operatorname{Inf}_{j}\left(A_{i}\right)$, each $m_{i j}^{\mathscr{A}}(\cdot)$ is defined by: 


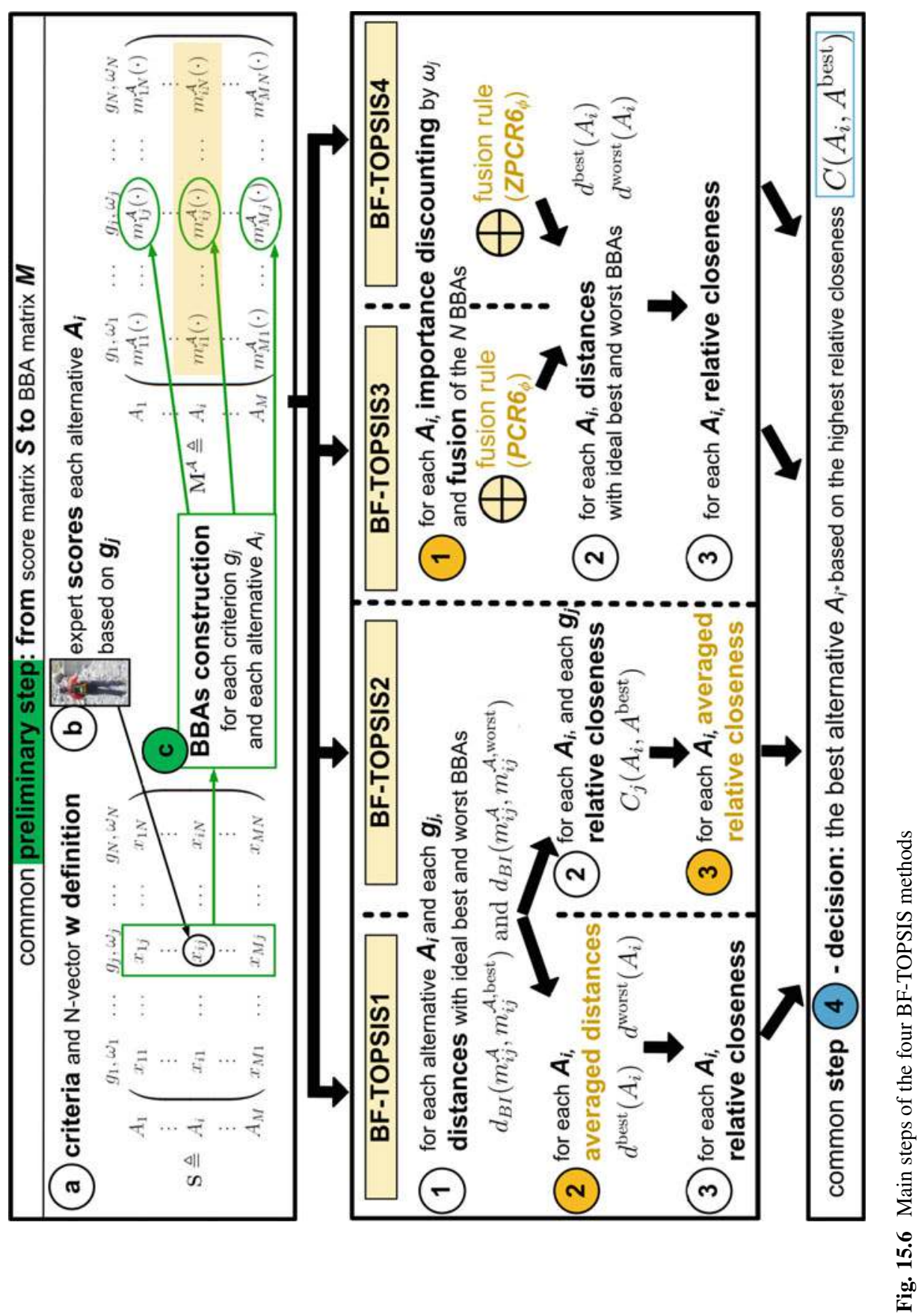




$$
\begin{gathered}
m_{i j}^{\mathscr{A}}\left(A_{i}\right) \triangleq \begin{cases}\frac{\operatorname{Sup}_{j}\left(A_{i}\right)}{A_{\text {max }}^{j}} & \text { if } A_{\text {max }}^{j} \neq 0 \\
0 & \text { if } A_{\text {max }}^{j}=0\end{cases} \\
m_{i j}^{\mathscr{A}}\left(\bar{A}_{i}\right) \triangleq \begin{cases}\frac{\operatorname{Inf}_{j}\left(A_{i}\right)}{A_{\text {min }}^{j}} & \text { if } A_{\text {min }}^{j} \neq 0 \\
0 & \text { if } A_{\text {min }}^{j}=0\end{cases} \\
m_{i j}^{\mathscr{A}}\left(A_{i} \cup \bar{A}_{i}\right) \triangleq m_{i j}^{\mathscr{A}}(\Theta) \triangleq 1-\left(\operatorname{Bel}_{i j}^{\mathscr{A}}\left(\bar{A}_{i}\right)+\operatorname{Bel}_{i j}^{\mathscr{A}}\left(A_{i}\right)\right)
\end{gathered}
$$

The four BF-TOPSIS methods differ from each other in the way they process the matrix $\mathbf{M}^{\mathscr{A}}$. All of them compute the relative closeness of each alternative $A_{i}$ with an ideal best solution $A^{\text {best }}$ denoted by $C\left(A_{i}, A^{\text {best }}\right)$. The preference ordering of all alternatives is built given the following criterion: the higher it is, the better $A_{i}$ is. An extension of BF-TOPSIS methods for dealing with imprecise scores is proposed in [48].

\subsubsection{BF-TOPSIS1}

(1) For each $A_{i}$ and $g_{j}$, the ideal best and worst BBAs are defined by $m_{i j}^{\text {best }}\left(A_{i}\right) \triangleq 1$ and $m_{i j}^{\text {worst }}\left(\bar{A}_{i}\right) \triangleq 1$ which are used to compute the distances $d_{B I}\left(m_{i j}^{\mathscr{A}}, m_{i j}^{\mathscr{A} \text {,best }}\right)$ and $d_{B I}\left(m_{i j}^{\mathscr{A}}, m_{i j}^{\mathscr{A} \text {,worst }}\right)$.

(2) The respective averaged distances $d^{\text {best }}\left(A_{i}\right)$ and $d^{\text {worst }}\left(A_{i}\right)$ are computed by weighting previous distances by importance weights $\omega_{j}$ of criteria $g_{j}$.

(3) For each $A_{i}$, the relative closeness is computed by:

$$
C\left(A_{i}, A^{\text {best }}\right) \triangleq \frac{d^{\text {worst }}\left(A_{i}\right)}{d^{\text {worst }}\left(A_{i}\right)+d^{\text {best }}\left(A_{i}\right)}
$$

\subsubsection{BF-TOPSIS2}

(1) This step is the same as for BF-TOPSIS1. (2) For each $A_{i}$ and $g_{j}$, the relative closeness $C_{j}\left(A_{i}, A^{\text {best }}\right)$ is computed. (3) The averaged relative closeness $C\left(A_{i}, A^{\text {best }}\right)$ is computed by weighting $C_{j}\left(A_{i}, A^{\text {best }}\right)$ by importance weights $\omega_{j}$ of criteria $g_{j}$.

\subsubsection{BF-TOPSIS3}

(1) For each $A_{i}$, the $N$ BBAs $m_{i j}^{\mathscr{A}}(\cdot)$ are combined through PCR6 fusion rule to give $m_{i}^{\mathscr{A}}(\cdot)$ taking into account the importance factor $\omega_{j}$ of each criterion $g_{j}$ [33]. (2) For 
each $A_{i}$, the ideal best and worst BBAs allow to give $d^{\text {best }}\left(A_{i}\right)=d_{B I}\left(m_{i}^{\mathscr{A}}, m_{i}^{\mathscr{A} \text {, best }}\right)$ and $d^{\text {worst }}\left(A_{i}\right)=d_{B I}\left(m_{i}^{\mathscr{A}}, m_{i}^{\mathscr{A} \text {,worst }}\right)$. (3) This step is the same as for BF-TOPSIS1.

\subsubsection{BF-TOPSIS4}

This method differs from BF-TOPSIS3 only by the choice of the ZPCR6 fusion rule [49] instead of PCR6 rule of combination.

\subsubsection{FCOWA-ER: Choice Under Epistemic Uncertainty}

Given the payoff matrix $\mathbf{C}$ defined by Eq. (15.2), the DMP requires choosing the best alternative $A_{i^{\star}} \in \mathscr{A}$. COWA-ER has been proposed [11] for such decision-making given uncertain knowledge on $\mathscr{S}$. It mixes cautiously the principle of Yager's Ordered Weighted Averaging (OWA) approach based on belief function theory [13] with fusion rules, notably the PCR6 one [30]. As detailed in Fig. 15.7, FCOWAER [12] is a modified version of COWA-ER using fuzzy sets which improves performances of COWA-ER and reduces its computational burden.

\subsubsection{From the OWA Approach...}

Under ignorance, Yager uses the OWA operator as a weighted average of ordered values of a variable defined by Eq. (15.21). For each $A_{i}, i=1, \ldots, M$, it consists in choosing a normalized set of weighting factors $\mathbf{W}_{\mathbf{i}}=\left[w_{i 1}, \ldots, w_{i k}, \ldots, w_{i K}\right]$, where $w_{i k} \in[0,1], \sum_{k} w_{i k}=1$, and $\mathbf{W}_{\mathbf{i}}$ depends on the DM attitude: $\mathbf{W}_{\mathbf{i}}=$ $[0,0, \ldots, 0,1]$ represents the pessimistic attitude, while $\mathbf{W}_{\mathbf{i}}=[1,0, \ldots, 0,0]$ is used for the optimistic one. The OWA value $V_{i}$ is computed for the collection of payoffs $C_{i 1}, C_{i 2}, \ldots, C_{i K}$, with $b_{i k}$ as the $k^{\text {th }}$ largest element in it. The best $A_{i^{\star}}$ is chosen with $i^{\star} \triangleq \arg \max _{i}\left\{V_{i}\right\}$.

Under epistemic uncertainty, considering the states of the nature $\mathscr{S}$ as the FoD, Yager represents the DM belief structure by a BBA $m^{\mathscr{S}}(\cdot): 2^{\mathscr{S}} \rightarrow[0,1]$, which is characterized by the $s$ focal elements $X_{r} \in 2^{\mathscr{S}}$. For each alternative $A_{i}$, restricting the states of the nature to $S_{k} \in X_{r}$, one has $\mathbf{M}_{\mathbf{i r}} \triangleq\left\{C_{i k} \mid S_{k} \in X_{r}\right\}, r=1, \ldots, s$. For each $A_{i}$, each $X_{r}$, and some DM attitude chosen a priori, the OWA value $V_{i r}=$ OWA $\left(\mathbf{M}_{\mathbf{i r}}\right)$ is computed. The derivation of a generalized expected value $C_{i}$ of payoff is defined by Eq. (15.22). The best $A_{i^{\star}}$ is thus chosen with $i^{\star} \triangleq \arg \max _{i}\left\{C_{i}\right\}$.

$$
\begin{gathered}
V_{i} \triangleq \operatorname{OWA}\left(C_{i 1}, C_{i 2}, \ldots, C_{i K}\right)=\sum_{k} w_{i k} \cdot b_{i k} \\
C_{i}=\sum_{r=1}^{s} m^{\mathscr{S}}\left(X_{r}\right) V_{i r}
\end{gathered}
$$




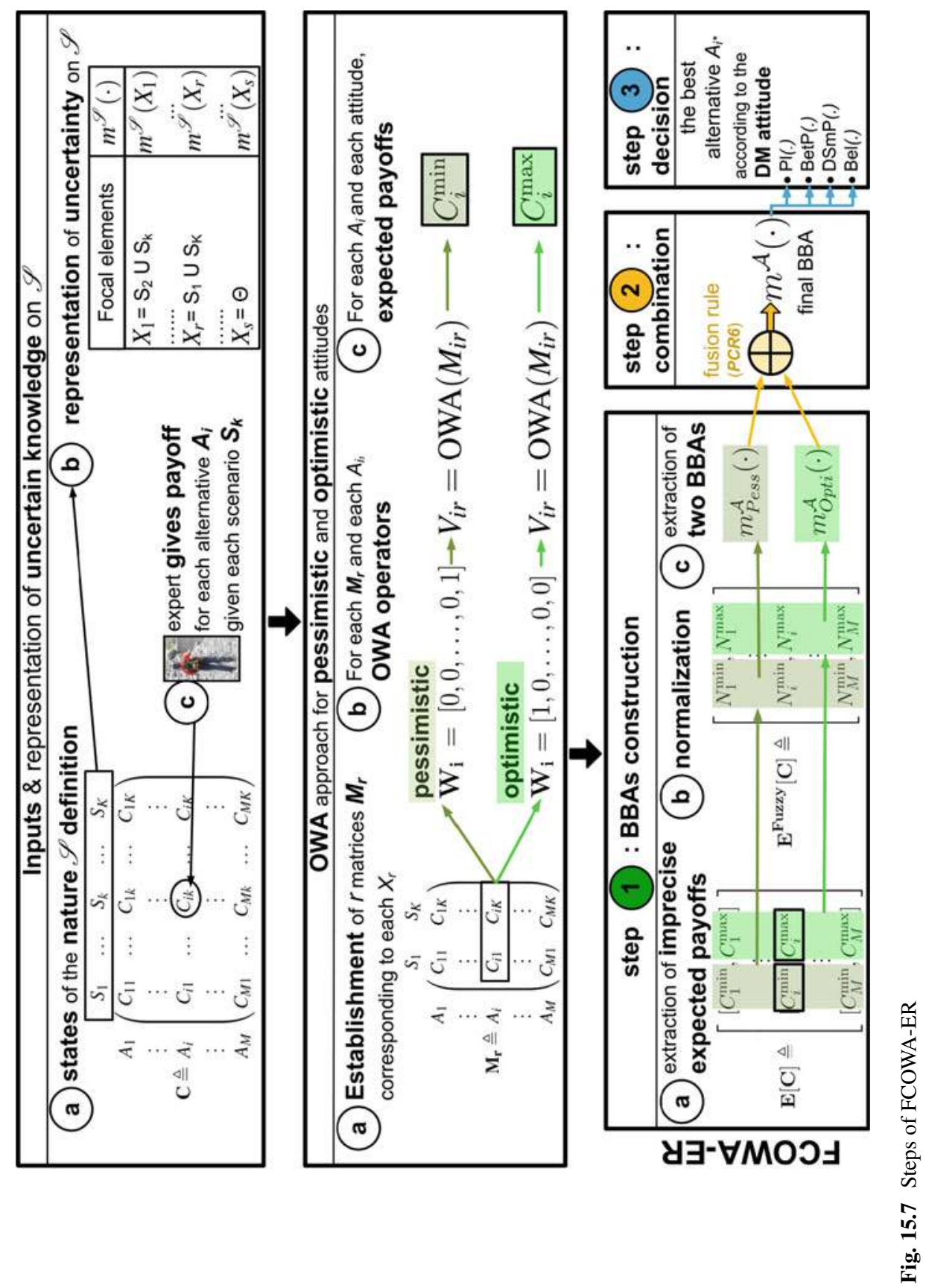




\subsubsection{2 …to the COWA-ER and FCOWA-ER Approaches}

For each alternative $A_{i}$, COWA-ER method exploits only the results of the two extreme attitudes (pessimistic and optimistic OWA) jointly [11]. Decision-making under uncertainty is thus based on the $M$ imprecise valuations (or intervals) of expected payoffs gathered in the $M$-vector $\mathbf{E}[\mathbf{C}]$ given by Eq. (15.23).

FCOWA-ER method [12] has been then developed to go beyond two COWA-ER limitations. (1) The BBAs obtained by using $\alpha$-cuts are consonant support (nested in order) without any correlation between information sources. (2) The computational time for making the combination does not depend on the number $M$ of alternatives.

\subsubsection{FCOWA-ER-Step 1: Construction of BBAs}

Each column in $\mathbf{E}[\mathbf{C}]$ is, respectively, normalized to obtain the column-wise normalized expected payoff $\mathbf{E}^{\text {Fuzzy }}[\mathbf{C}]$ given by Eq. (15.24).

$$
\begin{gathered}
\mathbf{E}[\mathbf{C}] \triangleq\left[\begin{array}{c}
{\left[C_{1}^{\min }, C_{1}^{\max }\right]} \\
{\left[C_{2}^{\min }, C_{2}^{\max }\right]} \\
\vdots \\
{\left[C_{M}^{\min }, C_{M}^{\max }\right]}
\end{array}\right] \\
\mathbf{E}^{\mathbf{F u z z y}}[\mathbf{C}] \triangleq\left[\begin{array}{c}
N_{1}^{\min }, N_{1}^{\max } \\
N_{2}^{\min }, N_{2}^{\max } \\
\vdots \\
N_{M}^{\min }, N_{M}^{\max }
\end{array}\right]
\end{gathered}
$$

The vectors $\mu_{1}=\left[N_{1}^{\min }, \ldots, N_{M}^{\min }\right]$ and $\mu_{2}=\left[N_{1}^{\max }, \ldots, N_{M}^{\max }\right]$ can be seen as two fuzzy membership functions (FMFs) $\mu: A_{i} \in \mathscr{A} \rightarrow[0,1]$. Given the FoD $\mathscr{A}=\left\{A_{1}, A_{2}, \ldots, A_{M}\right\}$, they are, respectively, converted into two BBAs $m_{\text {Pess }}^{\mathscr{A}}(\cdot)$ and $m_{\mathrm{Opti}}^{\mathscr{A}}(\cdot)$ using the $\alpha$-cut approach [50], considering $M$ as the number of $\alpha$-cuts.

\subsubsection{FCOWA-ER-Steps 2 and 3: Combination of the Two BBAs and Decision}

The two BBAs $m_{\text {Pess }}^{\mathscr{A}}(\cdot)$ and $m_{\text {Opti }}^{\mathscr{A}}(\cdot)$ are combined with the PCR6 fusion rule. The decision is about choosing $A_{i^{\star}}$ according to the DM attitude or the minimal distance. 


\subsection{Application to Efficiency of Torrential Protective Actions}

The example previously introduced in Fig. 15.2 is used to show how new belief function-based MCDAs can help DMs to decide on real DMPs. The DMP is first formalized. ER-MCDA, BF-TOPSIS, and FCOWA-ER are then successively applied without detailing computation steps: we only provide inputs and main results.

\subsubsection{Formalization of the Decision-Making Problem}

The problem is about comparing under uncertainty several torrential protective actions based on their efficiency by involving several criteria.

\subsubsection{Multi-criteria Decision-Making Problem}

Alternatives and decision criteria, with their scoring scales and importance weights, must be specified to provide the structure of the score matrix $\mathbf{S}$ defined by Eq. (15.1).

The set $\mathscr{A}$ gathers $M=5$ protective alternatives $A_{i}$ (Fig. 15.2):

- $A_{1}$ : doing nothing;

- $A_{2}$ : building check dam series in headwaters;

- $A_{3}$ : building a sediment trap on the alluvial fan apex;

- $A_{4}=A_{2} \cup A_{3}$ : building both check dam series and a sediment trap;

- $A_{5}$ : building individual protections for each element at-risk.

On the one hand, these actions aim at reducing potential damage on elements-atrisk. Several types of damage can occur such as housing destruction or environmental damage due to destruction of dangerous sites, etc. Their assessment in monetary value can strongly be debated as, for example, for human casualties [6]. On the other hand, each alternative involves high investment and maintenance cost.

A DM thus considers $N=5$ criteria $g_{j}$ with specific scoring scale $X_{j}$ [6] to compare alternatives according to their efficiency. He wants to minimize $g_{1}$ and $g_{2}$ (decreasing preference) and to maximize $g_{3}, g_{4}$, and $g_{5}$ (increasing preference), with:

- $g_{1}$ : investment cost in $€\left(x_{i 1} \in X_{1}=\mathbb{R}^{+}\right)$;

- $g_{2}$ : annual maintenance cost in $€\left(x_{i 2} \in X_{2}=\mathbb{R}^{+}\right)$;

- $g_{3}$ : annual risk reduction of damaged houses surface in $m^{2}\left(x_{i 3} \in X_{3}=\mathbb{R}^{+}\right)$;

- $g_{4}$ : annual risk reduction in human casualties $\left(x_{i 4} \in X_{4}=\mathbb{R}^{+}\right)$;

- $g_{5}$ : annual risk reduction in number of dangerous sites $\left(x_{i 5} \in X_{5}=\mathbb{R}^{+}\right)$. 
In practice, for each $A_{i}$ and $g_{j}$, annual risk reduction $\Delta R_{j}\left(A_{i}\right)$ is computed as reduction of potential damage expected value: $\Delta R_{j}\left(A_{i}\right)=R_{j}(0)-R_{j}\left(A_{i}\right)$, where $R_{j}(0)$ is the baseline risk (without $\left.A_{i}\right)$ and $R_{j}\left(A_{i}\right)$ is the residual risk with $A_{i}$ [6].

Given this set of criteria, DM uses the AHP process [3] to define the 5-vector of their importance weights: $\mathbf{w}=[0.08,0.04,0.10,0.46,0.32]$.

\subsubsection{Decision-Making Under Uncertainty}

Damage assessment depends on torrential hazards: without flood, there is no damage; during a big but rare flood, damage are higher; damage are much higher with a big debris flows. In practice, several scenarios of torrential hazards are thus taken into account to assess annual risk reduction criteria. They must be specified to provide the structure of the payoff matrix $\mathbf{C}$ defined by Eq. (15.2).

A DM considers a set of states of the nature $\mathscr{S}$ with $K=7$ scenarios as follows:

- liquid floods without bedload transport : $S_{1}$ with $Q_{l}<Q_{l 1}{ }^{4} ; S_{2}$ with $Q_{l} \geqslant Q_{l 1}$;

- floods with bedload transport: $S_{3}$ with $V_{s}<V_{s 1} ; S_{4}$ with $V_{s} \geqslant V_{s 1}$;

- debris flow : $S_{5}$ with $V_{l}<V_{l 1}{ }^{6} ; S_{6}$ with $V_{l 1} \leqslant V_{l}<V_{l 2} ; S_{7}$ with $V_{l} \geqslant V_{l 2}$

\subsubsection{ER-MCDA to Assign an Efficiency Label to Each Alternative}

In practice, a first DMP is about assigning each alternative to a qualitative efficiency label. Therefore, ER-MCDA methodology can be used.

\subsubsection{ER-MCDA Inputs}

FoD for decision gathers $n=4$ exhaustive and mutually exclusive efficiency labels, with $\Theta=\left\{\theta_{1}=\right.$ no, $\theta_{2}=$ low, $\theta_{3}=$ medium, $\theta_{4}=$ high $\}$. The mapping model of each criterion is provided in Fig. 15.8.

Two sources (experts) $s_{q}, q=1,2$, are assumed totally reliable $\left(\alpha_{1}=\alpha_{2}=1\right)$. For each $A_{i}$ and $g_{j}$, each one provides the possibility distributions $\pi_{q, i j}$ in Table 15.1.

\footnotetext{
${ }^{4} Q_{l}=$ liquid flow.

${ }^{5} V_{s}=$ solid volume.

${ }^{6} V_{l}=$ debris flow volume.
} 


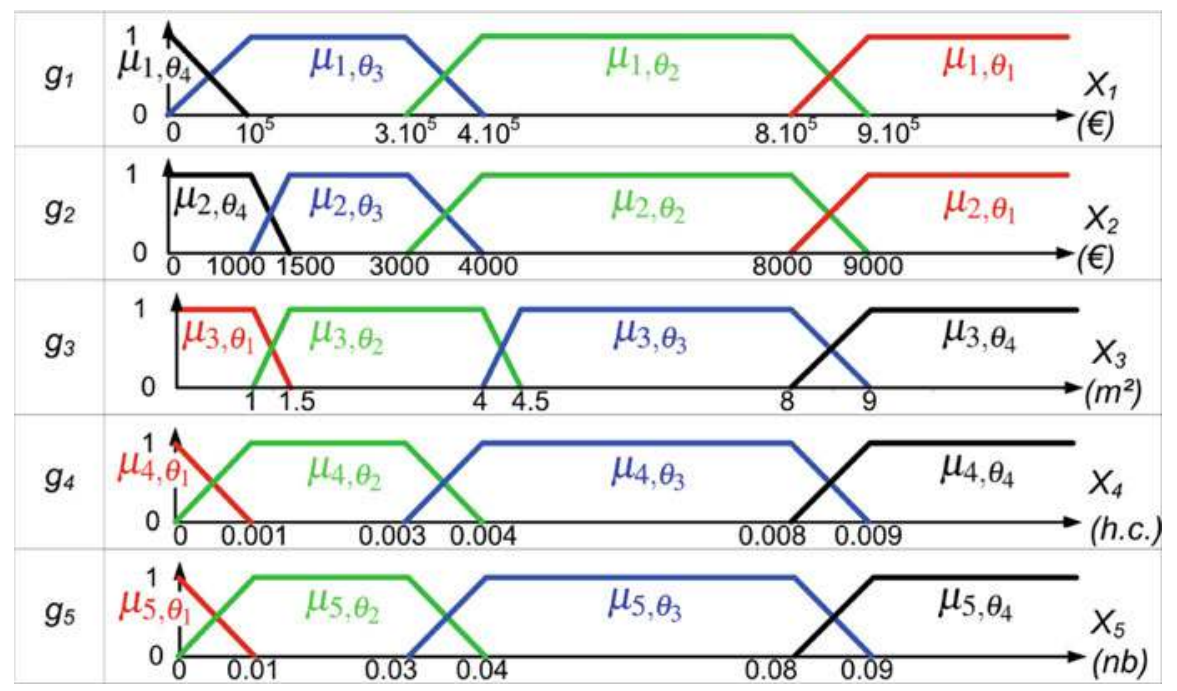

Fig. 15.8 ER-MCDA-input: $N=5$ mapping models

Table 15.1 ER-MCDA-input: imprecise evaluations $\pi_{q, i j}(q=1,2 ; i=1, \ldots, 5 ; j=1, \ldots, 5)$

\begin{tabular}{|c|c|c|c|c|c|c|}
\hline$A_{i}$ & $s_{q}$ & $g_{1}{ }^{a}$ & $g_{2}{ }^{a}$ & $g_{3}{ }^{b}$ & $g_{4}{ }^{\mathrm{b}}$ & $g_{5}{ }^{\mathrm{b}}$ \\
\hline$A_{1}$ & $s_{1}$ & $0,0,0,0$ & $0,0,0,0$ & 0,0 & 0,0 & 0,0 \\
\hline$A_{1}$ & $s_{2}$ & $0,0,0,0$ & $0,0,0,0$ & 0,0 & 0,0 & 0,0 \\
\hline$A_{2}$ & $s_{1}$ & $(2,3,3,5) \cdot 10^{5}$ & $(3,5,7,8) \cdot 10^{3}$ & $1.1,4.3$ & $(2.1,8.9) \cdot 10^{-3}$ & $(3.4,6.4) \cdot 10^{-2}$ \\
\hline$A_{2}$ & $s_{2}$ & $(1,2,2,3) \cdot 10^{5}$ & $(2,3,4,5) \cdot 10^{3}$ & $2,5.2$ & $(1.2,10.3) \cdot 10^{-3}$ & $(4.3,8.7) \cdot 10^{-2}$ \\
\hline$A_{3}$ & $s_{1}$ & $(2,4,4,6) \cdot 10^{5}$ & $(1,1.5,2,3) \cdot 10^{3}$ & $1.2,4.8$ & $(2.3,11.8) \cdot 10^{-3}$ & $(5.1,8.5) \cdot 10^{-2}$ \\
\hline$A_{3}$ & $s_{2}$ & $(4,5,6,8) \cdot 10^{5}$ & $(0.5,1,1,2) .10^{3}$ & $0.1,5.5$ & $(3.1,13.1) \cdot 10^{-3}$ & $(3.7,8.5) \cdot 10^{-2}$ \\
\hline$A_{4}$ & $s_{1}$ & $(4,7,7,11) \cdot 10^{5}$ & $(4,7.5,9,11) \cdot 10^{3}$ & $3.3,8.45$ & $(3.5,16.8) \cdot 10^{-3}$ & $(6.2,9.4) \cdot 10^{-2}$ \\
\hline$A_{4}$ & $s_{2}$ & $(5,7,8,11) \cdot 10^{5}$ & $(2.5,4,5,7) \cdot 10^{3}$ & $3.1,8.9$ & $(3.4,16.1) \cdot 10^{-3}$ & $(4.3,9.2) \cdot 10^{-2}$ \\
\hline$A_{5}$ & $s_{1}$ & $(9,10,12,14) \cdot 10^{5}$ & $0,0,0,0$ & $4.2,9.2$ & $(3.7,8.4) \cdot 10^{-3}$ & $(3.1,8.4) \cdot 10^{-2}$ \\
\hline$A_{5}$ & $s_{2}$ & $(8,9,10,11) \cdot 10^{5}$ & $0,0,0,0$ & $4.65,8.25$ & $(2 \cdot 1,9.3) \cdot 10^{-3}$ & $(1.5,9.2) \cdot 10^{-2}$ \\
\hline
\end{tabular}

${ }^{\mathrm{a} C}$ Criteria assessed by $\{\mathrm{a}, \mathrm{b}, \mathrm{c}, \mathrm{d}\}$ as shown in Fig. 15.3

${ }^{\mathrm{b}}$ Criteria assessed by $\{\mathrm{a}, \mathrm{d}\}(\mathrm{a}=\mathrm{b}$ and $\mathrm{c}=\mathrm{d})$

\subsubsection{ER-MCDA Results}

For each criterion $g_{j}$, the new mapping process is used in ER-MCDA-Step 2 to map each $\pi_{q, i j}$ of Table 15.1 into the $g_{j}$ mapping model of Fig. 15.8. It provides BBAs $m_{1, i j}^{\Theta}(\cdot)$ for $s_{1}$ and $m_{2, i j}^{\Theta}(\cdot)$ for $s_{2}$. Applying ER-MCDA-Step 3 on those BBAs, $M=5$ BBAs $m_{i}^{\Theta}(\cdot)$ are finally obtained in Table 15.2.

To avoid assumption $\left|\theta_{c}\right|=1$, ER-MCDA-Step 4 is based on computing distances $d_{B I}\left(m_{i}^{\Theta}, m_{X}^{\Theta}\right)$, from each column of Table 15.2. For each $A_{i}$, the chosen focal element $\hat{X}\left(A_{i}\right) \in 2^{\Theta}$, the corresponding value of minimal distance $d_{B I}^{\min }$, and the decision quality $q\left(\hat{X}\left(A_{i}\right)\right)$ are given in Table 15.3. 
Table 15.2 ER-MCDA-Step 3 results: BBAs $m_{i}^{\Theta}(\cdot)$ obtained for each alternative $A_{i}$

\begin{tabular}{l|l|l|l|l|l}
\hline Focal elements $X$ & $m_{1}^{\Theta}(\cdot)$ & $m_{2}^{\Theta}(\cdot)$ & $m_{3}^{\Theta}(\cdot)$ & $m_{4}^{\Theta}(\cdot)$ & $m_{5}^{\Theta}(\cdot)$ \\
\hline$\theta^{c}$ & 0.00049 & 0.00075 & 0.00067 & 0.00079 & 0.04062 \\
\hline$\theta_{1} \cup \theta^{c}$ & 0.96997 & 0 & 0 & 0 & 0.03157 \\
\hline$\theta_{2} \cup \theta^{c}$ & 0 & 0.26680 & 0.02393 & 0.02293 & 0 \\
\hline$\theta_{1} \cup \theta_{2} \cup \theta^{c}$ & 0 & 0 & 0 & 0.00830 & 0 \\
\hline$\theta_{3} \cup \theta^{c}$ & 0 & 0.36178 & 0.97036 & 0.71702 & 0.86412 \\
\hline$\theta_{2} \cup \theta_{3} \cup \theta^{c}$ & 0 & 0.36113 & 0.00416 & 0.02852 & 0 \\
\hline$\theta_{4} \cup \theta^{c}$ & 0.02954 & 0 & 0.00018 & 0.17687 & 0.03597 \\
\hline$\theta_{3} \cup \theta_{4} \cup \theta^{c}$ & 0 & 0.00954 & 0.00070 & 0.04557 & 0.02772 \\
\hline
\end{tabular}

Table 15.3 ER-MCDA-Step

4 results: decision based on $d_{B I}\left(m_{i}^{\Theta}, m_{X}^{\Theta}\right)$

\begin{tabular}{l|l|l|l}
\hline Alternative $A_{i}$ & $d_{B I}^{\min }$ & $\hat{X}\left(A_{i}\right)$ & $q\left(\hat{X}\left(A_{i}\right)\right)$ \\
\hline$A_{1}$ & 0.0172 & $\theta_{1} \cup \theta^{c}$ & 0.9990 \\
\hline$A_{2}$ & 0.1855 & $\theta_{2} \cup \theta_{3} \cup \theta^{c}$ & 0.9878 \\
\hline$A_{3}$ & 0.0154 & $\theta_{3} \cup \theta^{c}$ & 0.9991 \\
\hline$A_{4}$ & 0.1319 & $\theta_{3} \cup \theta^{c}$ & 0.9919 \\
\hline$A_{5}$ & 0.0567 & $\theta_{3} \cup \theta^{c}$ & 0.9968 \\
\hline
\end{tabular}

Solution $A_{1}$ of doing nothing is mainly no efficient. Alternative $A_{2}$ is lowly or mediumly efficient, while the three other ones are mediumly efficient. It corresponds to a partial preference order: $A_{3} \sim A_{4} \sim A_{5} \succeq A_{2} \succ A_{1}$.

As shown in Table 15.3, quality indicators of decisions are similar for $A_{3}$ and $A_{5}$ and better than for $A_{4}$. For $A_{2}$, global mass is more distributed with $m_{2}^{\Theta}\left(\theta_{3} \cup \theta^{c}\right)=$ $0.36, m_{2}^{\Theta}\left(\theta_{2} \cup \theta_{3} \cup \theta^{c}\right)=0.36$, and $m_{2}^{\Theta}\left(\theta_{2} \cup \theta^{c}\right)=0.27$ : decision quality is less good.

\subsubsection{BF-TOPSIS to Rank Alternatives}

Another DMP is about ranking all potential solutions given previous criteria. BFTOPSIS methods are compared to help to solve it.

\subsubsection{BF-TOPSIS Inputs}

A precise score of each $A_{i}$ based on each $g_{j}$ must be provided to obtain the score matrix $\mathbf{S}$, consistent with possibility distributions $\pi_{q, i j}$ given in Table 15.1 . To take into account decreasing preference, initial scores of $g_{1}$ and $g_{2}$ are multiplied by -1 providing the score matrix $\mathbf{S}^{\text {pref }}$ defined by Eq. (15.25). 
Table 15.4 BF-TOPSIS results: relative closeness $C\left(A_{i}, A^{\text {best }}\right)$

\begin{tabular}{l|l|l|l|l}
\hline Alternative $A_{i}$ & BF-TOPSIS1 & BF-TOPSIS2 & BF-TOPSIS3 & BF-TOPSIS4 \\
\hline$A_{1}$ & 0.12 & 0.12 & 0.03 & 0.03 \\
\hline$A_{2}$ & 0.49 & 0.49 & 0.68 & 0.68 \\
\hline$A_{3}$ & 0.66 & 0.66 & 0.85 & 0.85 \\
\hline$A_{4}$ & 0.69 & 0.69 & 0.88 & 0.88 \\
\hline$A_{5}$ & 0.92 & 0.92 & 0.97 & 0.97 \\
\hline
\end{tabular}

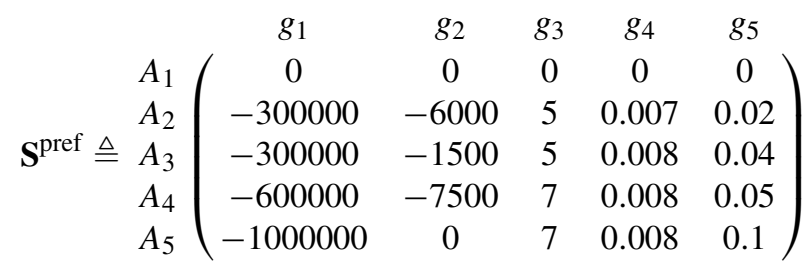

\subsubsection{BF-TOPSIS Results}

Given $\mathbf{S}^{\text {pref }}$, applying the four BF-TOPSIS methods provides relative closeness $C\left(A_{i}, A^{\text {best }}\right)$ of each alternative $A_{i}$ with the ideal best solution $A^{\text {best }}$ in Table 15.4.

In this case, whatever the BF-TOPSIS method used, preference ranking of all alternatives according to descending order of $C\left(A_{i}, A^{\text {best }}\right)$ is $A_{5} \succ A_{4} \succ A_{3} \succ$ $A_{2} \succ A_{1}$. In [6], for this application case, authors not only give computation details but also a comparison with classical MCDA methods such as CBA and AHP. They show that CBA is very sensitive to criteria choice for monetary valuation such as the human life $\left(g_{4}\right)$ and that BF-TOPSIS is more robust to rank reversal problems than AHP.

\subsubsection{FCOWA-ER to Choose the Best Alternative Under Uncertainty}

The final practical DMP is about choosing the best solution to implement, considering the knowledge on the states of the nature.

\subsubsection{FCOWA-ER Inputs}

For each $A_{i}, i=1, \ldots, 5$ and each scenario $S_{k}, k=1, \ldots, 7, C_{i k}$ is the efficiency level in the $5 \times 7$ matrix $\mathbf{C}$ defined by Eq. (15.26). It can be extracted after implementing ER-MCDA to solve a previous MCDM problem, given a specific $S_{k}$. A quantitative transformation of labels $\theta_{e}, e=1, \ldots, n$ into $[1 ; 10]$ (the higher 
Table 15.5 FCOWA-ER result: credibility, BetP, DSmP ${ }_{\epsilon=10^{-6}}$, and plausibility of $A_{i}$ efficiency

\begin{tabular}{l|l|l|l|l}
\hline$A_{i}$ & $\operatorname{Bel}^{\mathscr{A}}\left(A_{i}\right)$ & $\operatorname{BetP}^{\mathscr{A}}\left(A_{i}\right)$ & $\operatorname{DSmP}^{\mathscr{A}}\left(A_{i}\right)_{\epsilon=10^{-6}}$ & $\mathrm{Pl}^{\mathscr{A}}\left(A_{i}\right)$ \\
\hline$A_{1}$ & 0.000000 & 0.027908 & 0.000004 & 0.139530 \\
\hline$A_{2}$ & 0.000000 & 0.060282 & 0.000008 & 0.269030 \\
\hline$A_{3}$ & 0.000000 & 0.132010 & 0.000013 & 0.470130 \\
\hline$A_{4}$ & 0.000000 & 0.180390 & 0.000015 & 0.566890 \\
\hline$A_{5}$ & 0.404960 & 0.599420 & 0.999960 & 1.000000 \\
\hline
\end{tabular}

is score, the higher is payoff) is proposed. Results of ER-MCDA in Tables 15.2 and 15.3 help give payoffs for $S_{4}$.

$$
\mathbf{C}=\left[\begin{array}{rrrrrrr}
9 & 3 & 2 & 2 & 1 & 1 & 1 \\
10 & 8 & 4 & 3 & 2 & 1 & 1 \\
10 & 7 & 6 & 6 & 4 & 4 & 1 \\
10 & 6 & 8 & 7 & 3 & 2 & 1 \\
10 & 8 & 6 & 6 & 6 & 5 & 1
\end{array}\right]
$$

Expert represents uncertainty on the states of the nature $\mathscr{S}$ through a BBA $m^{\mathscr{S}}(\cdot)$. $s=4$ focal elements $X_{r} \in 2^{\mathscr{S}}$ are considered:

$m^{\mathscr{S}}\left(X_{1}\right)=m^{\mathscr{S}}\left(S_{1} \cup S_{3} \cup S_{5}\right)=0.4, m^{\mathscr{S}}\left(X_{2}\right)=m^{\mathscr{S}}\left(S_{2} \cup S_{4} \cup S_{5} \cup S_{6}\right)=0.25$, $m^{\mathscr{S}}\left(X_{3}\right)=m^{\mathscr{S}}\left(S_{7}\right)=0.1, m^{\mathscr{S}}\left(X_{4}\right)=m^{\mathscr{S}}(\mathscr{S})=0.25$.

$X_{1}, X_{2}$, and $X_{3}$ are partial ignorances, and $X_{4}$ is the full ignorance.

\subsubsection{FCOWA-ER Results}

Given payoff matrix $\mathbf{C}$ and BBA $m^{\mathscr{S}}(\cdot)$, applying FCOWA-ER steps, provides the two BBAs $m_{\text {Pess }}^{\mathscr{A}}(\cdot)$ and $m_{\text {Opti }}^{\mathscr{A}}(\cdot)$ for the FoD $\mathscr{A}$ (step 1) which are combined to give the final BBA $m_{\mathrm{PCR} 6}^{\mathscr{A}}(\cdot)$ through PCR6 fusion rule (step 2). Table 15.5 shows values of $\operatorname{Bel}^{\mathscr{A}}(\cdot), \operatorname{BetP}^{\mathscr{A}}(\cdot), \mathrm{DSmP}_{\epsilon=10^{-6}}^{\mathscr{A}}(\cdot)$ and $\mathrm{Pl}^{\mathscr{A}}(\cdot)$ based on $m_{\mathrm{PCR} 6}^{\mathscr{A}}(\cdot)$ (step 3).

Whatever the decision rule, the best action $A_{i^{\star}}$ is always $A_{5}$. The total preference ranking is deducted: $A_{5} \succ A_{4} \succ A_{3} \succ A_{2} \succ A_{1}$.

\subsection{Conclusions and Perspectives}

In practice, torrential risk managers must decide on the best action to reduce damage on elements-at-risk. Therefore, the comparison of efficiency of potential alternatives is generally used. Each one can be assessed through qualitative labels that require a partial ranking of alternatives, but it generally cannot help to choose the best action that requires a total preference ranking. 
The decisions are based on several criteria, such as costs and different types of damage reduction, imperfectly assessed under an epistemically uncertain environment corresponding to torrential hazards. Confronted with such difficulties, decisions are generally based on expert knowledge which directly takes into account imperfect information.

This chapter shows how recent developments of MCDAs based on belief function theory actually can help decision-makers in their decision process. A practical example is proposed and new methods, showing their possible combination for a global decision-making was applied.

Whatever the method is used, the first step of any DMP is to define alternatives to compare, criteria to take into account, their importance weights which represent the DM preference between them, and the set of states of the nature. Given the scores of each alternative based on each criterion, the final step is about aggregating this multi-criteria and multi-scenario evaluation to help decision.

The three methods applied in this chapter (ER-MCDA, BF-TOPSIS, FCOWAER) are combined at a combination step, while the PCR6 fusion rule is preferred. Nevertheless, analyzing the effect of this choice on the results of each method should be done.

To solve MCDM problems, ER-MCDA helps to take into account imperfect evaluation of criteria potentially provided by several sources. FoD of decision is first specified through qualitative or quantitative labels. Each expert is considered as source who gives a possibility distribution (imprecise scoring) and a mapping model based on fuzzy sets. ER-MCDA makes it possible to choose a label for each alternative providing the quality of this decision.

The four BF-TOPSIS methods help a total preference ranking of all alternatives with a better robustness to rank reversal problems than classical MCDAs. It is based on a precise score matrix representing the MCDM problem. Using intermediary results of ER-MCDA as an intermediary decision step helps to take into account imprecise scoring in BF-TOPSIS.

FCOWA-ER is different from the two previous methods because it proposes a method to solve DMP under uncertainty. It improves the OWA method used when the knowledge of the states of the nature is uncertain. It was first developed to decide given a precise scoring of each payoff. As for BF-TOPSIS, it is possible to apply ERMCDA for each scenario and to propose a quantitative transformation if qualitative labels are used. It thus helps to take into account initial imperfect scoring.

From an operational point of view, this chapter shows how theoretical methods can help a better formalization of the decision-making process. Indeed, expert and DM elicitation is always needed to express, given the DMP under concern, the criteria to take into account, their importance, and the preferences for their evaluation. Moreover, methods have been applied to DMPs related to protection efficiency, but they are generic and can be applied for any other DMP.

Acknowledgements This study was partially funded by the French Agricultural and Forest Ministry (MAA) and the French Environment Ministry (MTES). 


\section{References}

1. S. Carladous, G. Piton, A. Recking, F. Liébault, D. Richard, J.-M. Tacnet, D. Kuss, F. Philippe, Y. Quefféléan, O. Marco, Towards a better understanding of the today French torrents management policy through a historical perspective, in 3rd International Conference FLOODrisk, Lyon (2016)

2. B. Roy, Méthodologie Multicritère d'Aide à la Décision (Economica Collection Gestion, Paris, 1985)

3. T.L. Saaty, Multicriteria Decision Making - The Analytic Hierarchy Process: Planning, Priority Setting, Resource Allocation (McGraw-Hill, Pittsburgh, 1980)

4. Y.J. Lai, T.Y. Liu, C.L. Hwang, TOPSIS for MODM. Eur. J. Oper. Res. 76, 486-500 (1994)

5. N. Hanley, C.L. Spash, Cost-Benefit Analysis and the Environment (Edward Elgar, Cheltenham, 1993)

6. S. Carladous, J.-M. Tacnet, J. Dezert, D. Han, M. Batton-Hubert, Evaluation of efficiency of torrential protective structures with new BF-TOPSIS methods, in 19th International Conference on Information Fusion, Heidelberg (2016)

7. J.-M. Tacnet, M. Batton-Hubert, J. Dezert, A two-step fusion process for multi-criteria decision applied to natural hazards in mountains, in International Workshop on the Theory of Belief Functions, Brest (2010)

8. L.A. Zadeh, Fuzzy sets. Inf. Control 8, 338-353 (1965)

9. L.A. Zadeh, Fuzzy sets as a basis for a theory of possibility. Fuzzy Sets Syst. 1, 3-28 (1978)

10. J. Dezert, D. Han, H. Yin, A new belief function based approach for multi-criteria decisionmaking support, in 19th International Conference on Information Fusion, Heidelberg (2016)

11. J.-M. Tacnet, J. Dezert, Cautious OWA and evidential reasoning for decision making under uncertainty, in 14th International Conference on Information Fusion, Chicago (2011), pp. 2074-2081

12. D. Han, J. Dezert, J.-M. Tacnet, C. Han, A fuzzy-cautious OWA approach with evidential reasoning, in 15th International Conference on Information Fusion, Singapore (2012), pp. 278-285

13. R. Yager, Decision making under Dempster-Shafer uncertainties. Stud. Fuzziness Soft Comput. 219, 619-632 (2008)

14. F.H. Knight, Risk, Uncertainty, and Profit (Houghton Mifflin Company, 1921)

15. A. Schärlig, Décider sur plusieurs critères. Panorama de l'aide à la décision multicritère (Presses Polytechniques et Universitaires Romandes, Lausanne, 1985)

16. J. von Neumann, O. Morgenstern, Theory of Games and Economic Behavior (Princeton University Press, Princeton, 1944)

17. B. Mazzorana, J. Hübl, S. Fuchs, Improving risk assessment by defining consistent and reliable system scenarios. Nat. Hazards Earth Syst. Sci. 9, 145-159 (2009)

18. L.J. Savage, Foundations of Statistics (Wiley, New York, 1954)

19. J.-M. Tacnet, Prise en compte de l'incertitude dans l'expertise des risques naturels en montagne par analyse multicritères et fusion d'information. Ph.D. Thesis, ENSMSE: Saint-Étienne (2009)

20. K.J. Arrow, L. Hurwicz, An optimality criterion for decision-making under ignorance, in Uncertainty and Expectations in Economics, ed. by C.F. Carter, J.L. Ford, G.L.S. Shackle (Basil Blackwell, Oxford, 1972), pp. 1-11

21. M. Allais, The so-called Allais paradox and rational decisions under uncertainty, in Expected Utility Hypotheses and the Allais Paradox. Contemporary Discussions of the Decisions Under Uncertainty with Allais' Rejoinder, ed. by M. Allais, O. Hagen (Springer, New-York, 1979)

22. G. Shafer, A Mathematical Theory of Evidence (Princeton University Press, Princeton, 1976)

23. S. Carladous, M.-P. Michaud, J.-M. Tacnet, Q. Delvienne, A survey on protection work databases used at the Alpine space level: analysis of contents and state of the art related to protection work effectiveness assessment in START-it-uP (WP4). European START-it-uP project, Grenoble (2014) 
24. A. Martin, Cours sur La fusion d'informations (ENSIETA, Rennes, 2005)

25. D. Dubois, H. Prade, Fuzzy sets, probability and measurement. Eur. J. Oper. Res. 40, 135-154 (1989)

26. D. Dubois, H. Prade, Properties of measures of information in evidence and possibility theories. Fuzzy Sets Syst. 24, 161-182 (1987)

27. D. Dubois, H. Prade, Possibility Theory - An Approach to Computerized Processing of Uncertainty (Springer, Paris, 1988)

28. R.P. Srivastava, Decision making under ambiguity: a belief-function perspective. Arch. Control Sci. 6(XLII), 5-27 (1997)

29. P. Smets, Decision making in the TBM: the necessity of the pignistic transformation. Int. J. Approx. Reason. 38, 133-147 (2005)

30. F. Smarandache, J. Dezert, Advances and Applications of DSmT for Information Fusion, vols. 1-4 (American Research Press, 2004-2015). http://www.onera.fr/fr/staff/jean-dezert

31. P. Smets, The transferable belief model. Artif. Intell. 66, 191-234 (1994)

32. R.R. Yager, Hedging in the combination of evidence. J. Inf. Optim. Sci. 4, 73-81 (1983)

33. F. Smarandache, J. Dezert, J.-M. Tacnet, Fusion of Sources of Evidence with Different Importances and Reliabilities (Workshop on the Theory of Belief Functions, Brest, 2010)

34. A. Martin, A.-L. Jousselme, C. Osswald, Conflict measure for the discounting operation on belief functions, in 11th International Conference on Information Fusion, Cologne (2008), pp. 1003-1010

35. L.A. Zadeh, On the Validity of Dempster's Rule of Combination. Memo M79/24 (University of California, Berkeley, 1979)

36. A. Tchamova, J. Dezert, On the behavior of Dempster's rule of combination and the foundations of Dempster-Shafer theory, in 6th International Conference of Intelligent Systems, Sofia (2012), pp. 108-113

37. J. Dezert, A. Tchamova, On the validity of Dempster's fusion rule and its interpretation as a generalization of Bayesian fusion rule. Int. J. Intell. Syst. 29, 223-252 (2014)

38. A. Martin, C. Osswald, A new generalization of the proportional conflict redistribution rule stable in terms of decision, in Advances and Applications of DSmT for Information Fusion Collected Works, ed. by F. Smarandache, J. Dezert, vol. 2 (American Research Press, Rehoboth, 2006)

39. J. Dezert, D. Han, J.-M. Tacnet, S. Carladous, Decision-making with belief interval distance, in 4th International Conference on Belief Functions, Prague (2016)

40. A. Irpino, R. Verde, Dynamic clustering of interval data using a Wasserstein-based distance. Pattern Recogn. Lett. 29, 1648-1658 (2008)

41. D. Han, J. Dezert, Y. Yang, New distance measures of evidence based on belief intervals, in 3rd International Conference on Belief Functions, Oxford (2014), pp. 432-441

42. Y.-M. Wang, Y. Luo, On rank reversal in decision analysis. Math. Comput. Model. 49, 12211229 (2009)

43. Y.B. Shin, S.G. Lee, D. Chun, D. Chung, A critical review of popular multi-criteria decision making methodologies. Issues Inf. Syst. 14, 358-365 (2013)

44. J.-M. Tacnet, J. Dezert, M. Batton-Hubert, AHP and uncertainty theories for decision making using the ER-MCDA methodology, in International Symposium on AHP, Sorrento (2011)

45. S. Carladous, J.-M. Tacnet, J. Dezert, G. Dupouy, M. Batton-Hubert, A new ER-MCDA mapping for decision-making based on imperfect information, in 4th International Conference on Belief Functions, Prague (2016)

46. S. Carladous, Approche intégrée d'aide à la décision basée sur la propagation de l'imperfection de l'informaiton - application à l'efficacité des mesures de protection torrentielle. Ph.D. Thesis, ENSMSE: Saint-Étienne (2016) 
47. H. Yin, J. Lan, X.-R. Li, Measures for ranking estimation performance based on single or multiple performance metrics, in 16th International Conference on Information Fusion, Istanbul (2013)

48. J. Dezert, D. Han, J.-M. Tacnet, Multi-criteria decision-making with imprecise scores and BFTOPSIS, in 20th International Conference on Information Fusion, Xi' an (2017)

49. F. Smarandache, J. Dezert, Modified PCR rules of combination with degrees of intersections, in 8th International Conference on Information Fusion, Washington, DC (2015), pp. 2100-2107

50. M.C. Florea, A.-L. Jousselme, D. Grenier, E. Bossé, A critical review of popular multi-criteria decision making methodologies. Approximation techniques for the transformation of fuzzy sets into random sets. Fuzzy Sets Syst. 159, 270-288 (2008) 